\title{
Safety of heparin bridging therapy for transrectal ultrasound-guided prostate biopsy in patients requiring temporary discontinuation of antithrombotic agents
}

\author{
Itsuto Hamano', Shingo Hatakeyama1* ${ }^{10}$, Tohru Yoneyama², Yuki Tobisawa', Osamu Soma', \\ Teppei Matsumoto' ${ }^{\text {, Hayato Yamamoto' }}$, Atsushi Imai², Takahiro Yoneyama², Yasuhiro Hashimoto², \\ Takuya Koie ${ }^{1}$ and Chikara Ohyama ${ }^{1,2}$
}

\begin{abstract}
Background: Safety of heparin bridging therapy for transrectal ultrasound-guided prostate (TRUS) biopsy in patients requiring temporary discontinuation of antithrombotic therapy is unknown. This study aimed to assess the relationship between heparin bridging therapy and the incidence of complications after TRUS biopsy.

Methods: From January 2005 to November 2015, we performed 1307 consecutive TRUS biopsies on 1134 patients in our hospital. The patients were assigned to two groups: those without heparin bridging (the control group) and those with temporary discontinuation of antithrombotic agents with heparin bridging therapy (the bridging group). A 10-12-core TRUS biopsy was performed; the patients were evaluated for bleeding-related complications.
\end{abstract}

Results: Of 1134 patients, 1109 (1281 biopsies) and 25 (26 biopsies) were assigned to the control and bridging group, respectively. Patient background did not significantly differ between the control and bridging groups, except for age, history of diabetes, cardiovascular diseases, and CHADS2 scores. Compared with the control group, the bridging group showed a significantly higher rate of complication for any complication (35 vs. 8.3\%, $P<0.001$ ), bleedingrelated complications (27 vs. 4.4\%), and urinary tract infection (7.7 vs. 1.2\%). No thromboembolic event was observed in the present study. Multivariate logistic analysis showed that heparin bridging therapy was a significant risk factor for the incidence of any complication and bleeding-related complications.

Conclusions: Heparin bridging therapy with temporal discontinuation of antithrombotic agents may increase the risk of complications after TRUS biopsy. Further, large-scale studies are required to clarify the safety of heparin bridging therapy.

Keywords: Antithrombotic agents, Discontinuation, Heparin bridging, Prostate biopsy

\section{Background}

An estimated 106,032 transrectal ultrasound-guided prostate biopsies (TRUS biopsies) are performed annually in Japan (Kakehi and Naito 2008). TRUS biopsy is the current gold standard for diagnosing prostate cancer

\footnotetext{
*Correspondence: shingoh@hirosaki-u.ac.jp

1 Department of Urology, Hirosaki University Graduate School

of Medicine, 5 Zaifu-cho, Hirosaki 036-8562, Japan

Full list of author information is available at the end of the article
}

and is one of the most commonly performed procedures. The complications of TRUS biopsy have previously been described (Loeb et al. 2013; Nam et al. 2013; Pinkhasov et al. 2012; Rosario et al. 2012). The most frequent of these, which are usually self-limiting, is hematuria (14.781.5\%) (Giannarini et al. 2007; Ihezue et al. 2005; Kakehi and Naito 2008). Although the majority of patients have minor hematuria without complications, a few $(0.3-6.2 \%)$ can develop severe problems (Nam et al. 2013; Pinkhasov et al. 2012; Rosario et al. 2012). 
As the world's population ages rapidly, a relatively high proportion of patients are on prescribed medication for comorbidities; these medications include longterm antithrombotic therapy using low-dose aspirin, clopidogrel/ticlopidine, and warfarin. Several prospective studies support the safety of continuation of lowdose aspirin in TRUS biopsy (Giannarini et al. 2007; Maan et al. 2003). Conversely, no recommendation exists regarding the management of non-aspirin antithrombotic agents (warfarin or clopidogrel/ticlopidine) before a TRUS biopsy. Clinicians have three options for management, depending on the risk of thrombosis. First, antithrombotic agents can be continued during procedures associated with low rates of bleeding complications. Second, they can be discontinued for several days prior to the procedure and restarted thereafter. Third, they can be discontinued with bridging anticoagulation using heparin (heparin bridging therapy), administered during the sub-therapeutic window, in patients at high risk of thromboembolic events (Douketis et al. 2008; El-Hakim and Moussa 2010). However, there are few prospective studies regarding the relationship between TRUS biopsy and continuation of antithrombotic agents. Furthermore, the safety of short-term discontinuation of antithrombotic agents with heparin bridging therapy in TRUS biopsy has not been well documented. Therefore, we aimed to assess the safety of heparin bridging therapy for TRUS biopsy in patients requiring temporary discontinuation of antithrombotic agents in the present study.

\section{Methods}

\section{Patient selection and evaluation}

From January 2005 to November 2015, we performed 1307 TRUS-biopsies on 1134 patients in our hospital. We retrospectively investigated the relationship between heparin bridging therapy and the incidence of complications after TRUS biopsy and evaluated the prevalence of these complications in patients not receiving heparin bridging therapy. Patients were assigned to two groups: those without heparin bridging (the control group, $\mathrm{n}=1109 ; 1281$ biopsies) and those with temporary discontinuation of antithrombotic agents with heparin bridging therapy (the bridging group, $\mathrm{n}=25$; 26 biopsies). The control group included patients not administered any antithrombotic agents or those with temporary discontinuation of antithrombotic agents. Clinical indication of heparin bridging therapy was assessed by the physician who had medicated the patient with antithrombotic agents, according to the individual risks of thromboembolism. A 10- to 12-core TRUS biopsy was performed, and patients were surveyed to evaluate any complications and bleeding-related events. The incidence of complications and grades for each group was assessed by reference of medical charts, according to the Common Terminology Criteria for Adverse Events (version 4.0). Multivariate logistic regression analysis was performed to assess the risk factors for the incidence of complications (of any grade), including age, heparin bridging, presence of comorbidities (diabetes mellitus and hypertension), number of biopsy cores, and the CHADS2 score (congestive heart failure: 1 point; hypertension: 1 point; age 75 or older: 1 point; diabetes mellitus: 1 point; prior stroke or transient ischemic attack or thromboembolism: 2 points). The CHADS2 score is a clinical prediction rule for estimating the risk of stroke in patients with nonrheumatic atrial fibrillation that is a common and serious heart arrhythmia associated with thromboembolic stroke (Gage et al. 2001). To evaluate the accuracy and reliability of the CHADS2 score in clinical practice, we compared the international normalized ratio of prothrombin time (PT-INR) and CHADS2 score between heparin bridging and non-bridging heparin-administered patients.

\section{Statistical analysis}

Statistical analyses of the clinical data were performed using SPSS version 22.0 (SPSS Inc., Chicago, IL, USA) and GraphPad Prism 5.03 (GraphPad Software, San Diego, CA, USA). Categorical variables were reported as percentages and compared using the Fisher's exact test. Quantitative data were expressed as medians with quartiles 1 and 3 (Q1 and Q3). Differences between the groups were statistically compared using the Student's $t$ test for normal distribution or the Mann-Whitney $U$-test for non-parametric distribution. $P$ values $<0.05$ were considered statistically significant. Risk factors for any complications and bleeding-related events were identified using univariate and logistic regression multivariate analyses. Odds ratios (ORs) with $95 \%$ confidence intervals were calculated after concurrently controlling for potential confounders. Variables included in the models were age ( $>70$ years), history of type 2 diabetes (positive), history of hypertension (positive), prostate-specific antigen $(>7.8 \mathrm{ng} / \mathrm{mL})$, prostate cancer (positive), the number of biopsy cores (>10), use of antithrombotic agents (positive), the number of antithrombotic agents (2 or more), use of warfarin (positive), heparin bridging (positive), and the CHADS2 score ( 2 or higher). The median was used as a cut-off value in quantitative data.

\section{Ethics statement}

This study was performed in accordance with the ethical standards of the Declaration of Helsinki and approved by the ethics review board of Hirosaki University School of Medicine (Authorization Number: 2013-315). For this type of retrospective study, formal consent was not required. 


\section{Results}

Of 1134 patients, 1109 (1281 biopsies) and 25 (26 biopsies) were assigned to the control and bridging groups, respectively. The control group was consisted of the patients without any antithrombotic agents (1013 biopsies) and those with temporary discontinuation of antithrombotic agents (268 biopsies). The rates of any complications and bleeding-related complications in without any antithrombotic agents and those with temporary discontinuation of antithrombotic agents were 7.7 and $10 \%(P=0.314), 4.5$ and $3.7 \%(P=0.737)$, respectively. Because no significant differences were observed in complication rates between the groups, we integrated those patients in control group.

In the bridging group, the number of patients with oral antithrombotic agents were as follows; warfarin 13, low-dose aspirin 3 , clopidogrel 1 , dabigatran 1 , warfarin + low-dose aspirin 3, low-dose aspirin + ticlopidine 2 , warfarin + low-dose aspirin + ticlopidine 2 , and warfarin + low-dose aspirin + cilostazol + sarpogrelate 1 .

There were no significant differences in patient backgrounds between the groups, except for age, history of diabetes, use of antithrombotic argents, cardiovascular diseases, and CHADS2 scores (Table 1). Compared with the control group, the patients in the heparin bridging group were significantly older (70 vs. 74 years), had a higher prevalence of diabetes (11 vs. 27\%), and a higher CHADS2 score (0 vs. 2 points). The total number of complications and the bleeding-related events were 106 $(8.3 \%)$ and $57(4.4 \%)$ cases in the control group and 9 (35\%) and 7 (27\%) cases in the bridging group, respectively. The incidence of complications in the bridging group was significantly higher than that in the control group. However, no significant difference was observed for severe (grade 3 or higher) complications. No thromboembolism-associated complication was observed in the present study (Table 1). Multivariate logistic analysis showed that diabetes, numbers of biopsy cores $>10$, and heparin bridging were selected as risk factors for the incidence of any complications (Table 2). In bleeding-related events, age $>70$ and heparin bridging were significant risk factors. Use of non-aspirin antithrombotic agents was not statistically significant for bleeding-related complications (Table 2).

Our study included 68 patients who were taking warfarin before TRUS biopsy. Of these, 19 and 49 patients were assigned to warfarin discontinuation with and without heparin bridging (discontinuation alone) groups by the primary physician, respectively. We compared the PT-INR and CHADS2 scores between these two groups. Our data showed that PT-INR was significantly higher in heparin bridging patients. However, CHADS2 score was not significantly different between the groups (Table 3).
To assess the safety of heparin bridging therapy, we compared patients with temporary discontinuation of antithrombotic agents $(\mathrm{n}=268)$ and the bridging group $(n=26)$. Although there were age differences between the groups, the rates of any complications and bleedingrelated complications in those with discontinuation and the bridging group are 9.7 and $35 \%(P=0.001), 3.7$ and $27 \%(P=0.001)$, respectively (Table 4$)$.

\section{Discussion}

The potential risk of severe hemorrhage requiring hospitalization was reportedly $1 / 500$ in men undergoing TRUS biopsy without warfarin administration, with a theoretical fivefold increase in men administered warfarin as an anticoagulant (Ramachandran et al. 2005). When a patient is considered to be at a low or intermediate risk for thromboembolism, most clinicians advocate discontinuation of antithrombotic therapy prior to TRUS biopsy and recommend continuation only after any bleeding has stopped (Cochlin 2005). The risk of thrombotic events associated with stopping warfarin is reportedly approximately 1\% (Wahl 1998; Yasaka et al. 2001). Several observational studies have suggested that simply interrupting warfarin therapy for $<7$ days is associated with a very low rate of thromboembolism and severe bleeding complications (Garcia et al. 2008; Wysokinski et al. 2008). A survey among urologists and radiologists found that $84 \%$ of urologists stopped warfarin 4 days before TRUS biopsy, and $95 \%$ of radiologists stopped it 5 days before TRUS biopsy (Connor and Wingate 1999). A PT-INR of less than 1.5 is accepted for most elective procedures (Kearon and Hirsh 1997).

When patients have a high risk of thromboembolism, either continuation of antithrombotic agents or discontinuation of antithrombotic agents with heparin bridging therapy is required. As a definitive recommendation regarding the management of antithrombotic agents before TRUS biopsy is yet to be established, the benefits and adverse effects of heparin bridging therapy are debatable. Clinicians have traditionally discontinued antithrombotic agents and started heparin bridging therapy, depending on the risk of thrombotic events (Douketis et al. 2008; El-Hakim and Moussa 2010). The guidelines for the management of antithrombotic agents for endoscopic procedures suggest heparin bridging therapy for higher-risk procedures (e.g., polypectomy) in high-risk patients (e.g., those with atrial fibrillation with mechanical valves, valvular heart disease, and/or a history of cerebrovascular accidents, and those who experience transient ischemic attacks) (Murasaki 2011). A recent prospective study suggested that heparin bridging therapy increased the risk of bleeding complications from interruption alone $(0.8 \%)$ to bridging 
Table 1 Patient background, and incidents and rates of complications

\begin{tabular}{|c|c|c|c|c|}
\hline & All & Control & Bridging & $P$ value \\
\hline Biopsy, n & 1307 & 1281 & 26 & \\
\hline Patients, $\mathrm{n}$ & 1134 & 1109 & 25 & \\
\hline Age (years) & $70(65,74)$ & $70(65,70)$ & $74(71,76)$ & $<0.001$ \\
\hline PSA (ng/mL) & $7.8(5.4,13.5)$ & $7.8(5.4,13.5)$ & $10.5(6.8,14.6)$ & 0.099 \\
\hline Diabetes, $\mathrm{n}$ & $131(13 \%)$ & $124(11 \%)$ & $7(27 \%)$ & 0.011 \\
\hline Hypertension, n & $423(32 \%)$ & $411(37 \%)$ & $12(46 \%)$ & 0.141 \\
\hline Frequencies of biopsy & $1(1,2)$ & $1(1,2)$ & $1(1,1)$ & 0.970 \\
\hline Number of cores & $10(10,12)$ & $10(10,12)$ & $10(10,10)$ & 0.523 \\
\hline Prostate cancer, $\mathrm{n}$ & $607(46 \%)$ & $594(46 \%)$ & $13(50 \%)$ & 0.843 \\
\hline Use of any antithrombotic agents (any), $n$ & $294(23 \%)$ & $268(21 \%)$ & $26(100 \%)$ & $<0.001$ \\
\hline Low dose aspirin & & $191(15 \%)$ & $11(42 \%)$ & \\
\hline Warfarin & & $52(4.1 \%)$ & $19(73 \%)$ & \\
\hline Clopidogel/ticlodipine & & $37(2.9 \%)$ & $7(27 \%)$ & \\
\hline NOAC & & $3(0.2 \%)$ & $1(3.8 \%)$ & \\
\hline \multicolumn{5}{|l|}{ Reason for antithrombotic agents use, $n$} \\
\hline Atrial fibrillation (Af) & $157(12 \%)$ & $139(11 \%)$ & $18(69 \%)$ & \\
\hline Myocardial infarction (MI) & $52(4.0 \%)$ & $46(3.6 \%)$ & $6(23 \%)$ & \\
\hline Others & $86(6.6 \%)$ & $84(6.6 \%)$ & $2(8 \%)$ & \\
\hline CHADS2 score & $0(0,0)$ & $0(0,1)$ & $2(0,2)$ & $<0.001$ \\
\hline \multicolumn{5}{|l|}{ Complications } \\
\hline All events, $\mathrm{n}$ & $118(9.0 \%)$ & $106(8.3 \%)$ & $9(35 \%)$ & $<0.001$ \\
\hline Grade 1 or 2 & $106(8.1 \%)$ & $95(7.4 \%)$ & $8(31 \%)$ & \\
\hline Grade 3 & $12(0.9 \%)$ & $11(0.9 \%)$ & $1(3.8 \%)$ & \\
\hline Bleeding related events, $\mathrm{n}$ & $64(4.9 \%)$ & $57(4.4 \%)$ & $7(27 \%)$ & $<0.001$ \\
\hline Grade 1 or 2 & $57(4.4 \%)$ & $51(4.0 \%)$ & $6(23 \%)$ & \\
\hline Grade 3 & $7(0.5 \%)$ & $6(0.5 \%)$ & $1(3.8 \%)$ & \\
\hline Urinary tract infection, $\mathrm{n}$ & $17(1.3 \%)$ & $15(1.2 \%)$ & $2(7.7 \%)$ & 0.043 \\
\hline Others, $\mathrm{n}$ & $34(2.6 \%)$ & $34(2.7 \%)$ & $0(0 \%)$ & 1.000 \\
\hline Thrombotic events, $n$ & $0(0 \%)$ & $0(0 \%)$ & $0(0 \%)$ & \\
\hline
\end{tabular}

Median and interquartile range (Q1, Q3) was used for consecutive variables

PT-INR prothrombin time-international normalized ratio, NOAC novel oral anticoagulant

(13\%) in colonoscopy and oral and ophthalmic surgeries (Garcia et al. 2008). However, there is little reliable evidence for the benefits and harm associated with heparin bridging therapy in TRUS biopsy. To our knowledge, this is the first report to describe the risk of heparin bridging therapy for TRUS biopsy in patients requiring temporary discontinuation of antithrombotic agents. Our data suggested that discontinuation of antithrombotic agents with heparin bridging therapy increased the incidence of complications after TRUS biopsy. Furthermore, there was an increase in bleeding-related complications and urinary tract infections. Although the majority of complication grades were low (1 or 2 ), the incidence rate of complications was significantly higher in heparin bridging patients than in control patients. Similarly to a previous study (Garcia et al. 2008), our results suggested the possibility that heparin bridging therapy may cause more harm than good. From a different perspective, this is reasonable because patients who are indicated for heparin bridging therapy have a number of severe comorbidities. In fact, our patients treated with heparin bridging therapy had a significantly higher prevalence of diabetes and cardiovascular diseases. Therefore, it is possible that those patients who were recommended heparin bridging therapy have a high risk for complications, and careful management of such complications is required in these patients.

In addition, the patients with temporary discontinuation of antithrombotic agents should be compared to the bridging group to assess the safety of heparin bridging therapy. The rates of any complications and bleeding-related complications in those with discontinuation and the bridging group were 9.7 and $35 \%(P=0.001)$, 3.7 and $27 \%(P=0.001)$, respectively. This result implies that the heparin bridging itself, in spite of the past use of 
Table 2 Uni- and multivariate logistic analysis of risk factors for complications

\begin{tabular}{|c|c|c|c|c|c|c|c|}
\hline & \multirow[t]{2}{*}{ Risk factors } & \multicolumn{3}{|c|}{ Any complications } & \multicolumn{3}{|c|}{ Bleeding-related events } \\
\hline & & $P$ value & HR & $95 \% \mathrm{Cl}$ & $P$ value & HR & $95 \% \mathrm{Cl}$ \\
\hline \multicolumn{8}{|l|}{ Univariate } \\
\hline Age & $>70$ years & 0.023 & 1.57 & $1.06-2.32$ & 0.004 & 2.14 & $1.27-3.61$ \\
\hline Diabetes & Positive & 0.004 & 2.09 & $1.26-3.44$ & 0.052 & 1.91 & $0.99-3.67$ \\
\hline Hypertension & Positive & 0.883 & 1.03 & $0.69-1.53$ & 0.802 & 0.94 & $0.55-1.58$ \\
\hline PSA & $>7.8 \mathrm{ng} / \mathrm{mL}$ & 0.641 & 0.91 & $0.62-1.34$ & 0.454 & 0.82 & $0.50-1.37$ \\
\hline Prostate cancer & Positive & 0.641 & 0.92 & $0.63-1.33$ & 0.859 & 0.96 & $0.59-1.55$ \\
\hline Number of biopsy cores & $>10$ cores & 0.063 & 1.48 & $0.98-2.23$ & 0.521 & 1.20 & $0.69-2.08$ \\
\hline Use of oral antithrombotic agents & Positive & 0.025 & 1.62 & $1.06-2.47$ & 0.425 & 1.26 & $0.71-2.23$ \\
\hline Number of oral antithrombotic agents & 2 or more & 0.043 & 1.38 & $1.01-1.90$ & 0.281 & 1.26 & $0.83-1.92$ \\
\hline Use of warfarin & Positive & 0.679 & 1.19 & $0.53-2.65$ & 0.745 & 1.19 & $0.42-3.37$ \\
\hline Heparin-bridging & Positive & 0.000 & 5.99 & $2.61-13.8$ & 0.000 & 7.91 & $3.20-19.6$ \\
\hline CHADS2 score & 2 or higher & 0.005 & 2.30 & $1.29-4.08$ & 0.003 & 2.83 & $1.42-5.61$ \\
\hline \multicolumn{8}{|l|}{ Multivariate } \\
\hline Age & $>70$ years & 0.108 & 1.39 & $0.93-2.09$ & 0.015 & 1.96 & $1.14-3.36$ \\
\hline Diabetes & Positive & 0.023 & 1.99 & $1.10-3.59$ & 0.243 & 1.60 & $0.73-3.52$ \\
\hline Number of biopsy cores & $>10$ cores & 0.025 & 1.62 & $1.06-2.47$ & 0.371 & 1.29 & $0.74-2.27$ \\
\hline Use of oral antithrombotic agents & Positive & 0.473 & 1.19 & $0.74-1.92$ & 0.280 & 0.68 & $0.33-1.38$ \\
\hline Heparin-bridging & Positive & 0.002 & 4.78 & $1.77-13.0$ & 0.001 & 7.52 & $2.29-24.8$ \\
\hline CHADS2 score & 2 or higher & 0.912 & 0.96 & $0.44-2.06$ & 0.655 & 1.25 & $0.47-3.31$ \\
\hline
\end{tabular}

Table 3 Background of patient who are taking warfarin

\begin{tabular}{llll}
\hline & \multicolumn{2}{c}{ Discontinuation of warfarin } & P value \\
\cline { 2 - 3 } & Without bridging & With bridging & \\
\hline Biopsy, $\mathrm{n}$ & 49 & 19 & \\
Patients, $\mathrm{n}$ & 46 & 18 & 0.406 \\
Age (years) & $72(68,76)$ & $73(71,76)$ & 0.333 \\
PSA (ng/mL) & $8.8(5.4,13.6)$ & $10.5(7.0,14.9)$ & 0.128 \\
Diabetes, $\mathrm{n}$ & $5(10 \%)$ & $5(26 \%)$ & 0.588 \\
Hypertension, $\mathrm{n}$ & $23(47 \%)$ & $7(37 \%)$ & 1.000 \\
Concurrent antithrom- & & & \\
botic agents & & $13(68 \%)$ & 0.026 \\
Warfarin alone, $\mathrm{n}$ & $34(69 \%)$ & $6(32 \%)$ & 0.701 \\
Warfarin + others, $\mathrm{n}$ & $15(31 \%)$ & $1.89(1.67,2.32)$ & \\
PT-INR (at a target level) & $1.55(1.19,1.92)$ & $1(0,3)$ & \\
CHAD2 score & $1(1,3)$ & &
\end{tabular}

Median and interquartile range $(\mathrm{Q} 1, \mathrm{Q} 3)$ was used for consecutive variables. PT-INR: prothrombin time-international normalized ratio

antithrombotic agents, affects the complication rate. Furthermore, the patient background of these two groups is compared to consider the influence of heparin bridging. The comorbidity rates of diabetes and hypertension in those with discontinuation and the bridging group were 15 and $27 \%(P=0.160)$, and 49 and $46 \%(P=0.839)$, respectively. This similarity of background also supports the significance of heparin bridging for complication rates.

We observed no thrombotic event in this cohort. Our results may be biased by the limitations of sample size and/or the ethnic differences. The risk of thrombotic disease is different between Japanese and Caucasians (Murasaki 2011). Therefore, we could not establish the benefits or adverse effects of heparin bridging therapy in this present study.

To balance the potential benefits and adverse effects, continuation of antithrombotic agents is another option for reducing thrombotic events. Several studies have suggested the safety of continuation of warfarin amid TRUS- biopsy. In the studies of Ihezue et al. (2005) and Chowdhury et al. (2012), no significant differences were observed in the severity of bleeding between the patients taking warfarin (36.7 and $27.9 \%$, respectively) and the controls (60.2 and $37.0 \%$, respectively). These results suggest that discontinuation of warfarin before TRUS biopsy is unnecessary. However, the limitations of these studies include the non-randomized design, potentially life-threatening hemorrhagic complications that may have been avoided only due to the sample size and limited information regarding the proper control of PT-INR. Another alternative is a switch of novel oral anticoagulant (NOAC). It may decrease the risk of bleeding and thromboembolism (Douketis et al. 2014). However, there 
Table 4 Comparison between patients with temporary discontinuation of antithrombotic agents and the bridging group

\begin{tabular}{|c|c|c|c|}
\hline & $\begin{array}{l}\text { Temporary discontinuation } \\
\text { of antithrombotic agents }\end{array}$ & Bridging & $P$ value \\
\hline Biopsy, n & 268 & 26 & \\
\hline Patients, n & 235 & 25 & \\
\hline Age (years) & $72(68,75)$ & $74(71,76)$ & 0.016 \\
\hline $\mathrm{PSA}(\mathrm{ng} / \mathrm{mL})$ & $7.6(5.3,13.4)$ & $10.5(6.8,14.6)$ & 0.329 \\
\hline Diabetes, n & $41(15 \%)$ & $7(27 \%)$ & 0.160 \\
\hline Hypertension, n & $132(49 \%)$ & $12(46 \%)$ & 0.839 \\
\hline Frequencies of biopsy & $1(1,1)$ & $1(1,1)$ & 0.124 \\
\hline Number of cores & $10(10,10)$ & $10(10,10)$ & 0.526 \\
\hline Prostate cancer, $\mathrm{n}$ & $131(49 \%)$ & $13(50 \%)$ & 0.913 \\
\hline Use of any antithrombotic agents (any), $n$ & $268(100 \%)$ & $26(100 \%)$ & \\
\hline Low dose aspirin & $191(15 \%)$ & $11(42 \%)$ & \\
\hline Warfarin & $52(4.1 \%)$ & $19(73 \%)$ & \\
\hline Clopidogel/ticlodipine & $37(2.9 \%)$ & $7(27 \%)$ & \\
\hline NOAC & $3(0.2 \%)$ & $1(3.8 \%)$ & \\
\hline Reason for antithrombotic agents use, $\mathrm{n}$ & & & $<0.001$ \\
\hline Atrial fibrillation (Af) & $139(52 \%)$ & $18(69 \%)$ & \\
\hline Myocardial Infarction (MI) & $46(17 \%)$ & $6(23 \%)$ & \\
\hline Others & $84(31 \%)$ & $2(8 \%)$ & \\
\hline CHADS2 score & $0(0,1)$ & $2(0,2)$ & $<0.001$ \\
\hline \multicolumn{4}{|l|}{ Complications } \\
\hline All events, $\mathrm{n}$ & $26(9.7 \%)$ & $9(35 \%)$ & 0.001 \\
\hline Grade 1 or 2 & $22(8.2 \%)$ & $8(31 \%)$ & \\
\hline Grade 3 & $4(1.5 \%)$ & $1(3.8 \%)$ & \\
\hline Bleeding related events, $\mathrm{n}$ & $10(3.7 \%)$ & $7(27 \%)$ & $<0.001$ \\
\hline Grade 1 or 2 & $8(2.9 \%)$ & $6(23 \%)$ & \\
\hline Grade 3 & $2(0.7 \%)$ & $1(3.8 \%)$ & \\
\hline Urinary tract infection, $\mathrm{n}$ & $4(1.5 \%)$ & $2(7.7 \%)$ & 0.002 \\
\hline Others, n & $12(4.5 \%)$ & $0(0 \%)$ & 1.000 \\
\hline Thrombotic events, $\mathrm{n}$ & $0(0 \%)$ & $0(0 \%)$ & \\
\hline
\end{tabular}

Median and interquartile range $(\mathrm{Q} 1, \mathrm{Q} 3)$ was used for consecutive variables

NOAC novel oral anticoagulant

is limited evidence for invasive procedures with interruption of NOAC administration. Further studies are needed to evaluate the benefits and adverse effects of discontinuation alone, continuation, or discontinuation with administration of heparin bridging therapy in TRUS biopsy.

To carefully assess the benefits and adverse effects, risk classification is necessary. One of the major risk classifications is the CHADS2 score (Gage et al. 2004). It is used to determine whether or not antithrombotic treatment is required. The CHADS2 score is simple and its applicability has been validated by many studies. Based on the CHADS2 score, this decision pertaining to the treatment modality should be discussed with the patient and the primary physician managing the antithrombotic agents. To assess the accuracy and reliability of CHADS2 scores in clinical practice, we selected 68 patients who were taking warfarin and compared the PT-INR and CHADS2 scores between patients with and without heparin bridging therapy. Our data showed that the target level of PTINR was significantly higher in patients with heparin bridging therapy. However, no relationship was observed between the CHADS2 score and a recommendation for heparin bridging therapy. This finding suggests the difficulty in truly defining risk stratification and decision making, and highlights the urgent need for large-scale randomized clinical trials to evaluate the management of continuation of antithrombotic agents or bridging therapy in patients undergoing TRUS biopsy.

The present study has several limitations, including small sample size, its retrospective nature, and the indication of heparin bridging therapy. Among these, the retrospective design may result in remarkable biases in 
this study. For instance, propensity score matching was not applied because of the small sample size. On the other hand, because the judgement regarding antithrombotic therapy possibly causes fatal complications such as intracranial hemorrhage and pulmonary embolism, the prospective study needs to be designed very carefully in the future. Although our study suggested that heparin bridging therapy may increase the risk of bleeding complications, safety of this therapy remains inconclusive. Despite these limitations, this study is the first to report the safety of heparin bridging therapy for TRUS biopsy in patients requiring temporary discontinuation of antithrombotic agents (Additional file 1).

\section{Conclusion}

In conclusion, bridging of temporal discontinuation of antithrombotic agents with heparin may increase the risk of complications. However, the limited number of patients with heparin bridging therapy obfuscates a clear interpretation of the results. Further large-scale studies are necessary to clarify the benefits and adverse effects of heparin bridging therapy.

\section{Additional file}

Additional file 1. Patient background and adverse events of all 1307 prostate biopsies.

\section{Abbreviations}

TRUS biopsy: transrectal ultrasound-guided prostate biopsy; ASA: acetylsalicylic acid; AEs: adverse events; PT-INR: prothrombin time-international normalized ratio; NOAC: non-vitamin K antagonist oral anticoagulation.

\section{Authors' contributions}

$\mathrm{IH}$; collection of data, analyzed of data, writing of the main article. SH; analyzed the data, writing of the main article. TY, YT, OS, TM, HY, Al, TY, YH; collection of data. TK; intellectual contribution to the manuscript. $\mathrm{SH}, \mathrm{CO}$; designed the plan of the study. All authors read and approved the final manuscript.

\section{Author details}

${ }^{1}$ Department of Urology, Hirosaki University Graduate School of Medicine, 5 Zaifu-cho, Hirosaki 036-8562, Japan. ${ }^{2}$ Department of Advanced Transplant and Regenerative Medicine, Hirosaki University Graduate School of Medicine, Hirosaki, Japan.

\section{Acknowledgements \\ The authors thank Dr. Yuki Fujita for the invaluable help with scientific suggestions.}

\section{Competing interests}

The authors declare that they have no competing interests, and no financial conflict of interests.

\section{Ethics approval and consent to participate}

This study was performed in accordance with the ethical standards of the Declaration of Helsinki and approved by the ethics review board of Hirosaki University School of Medicine (Authorization Number: 2013-315). For this type of retrospective study, formal consent was not required.

\section{Funding}

This work was supported by Grants-in-Aid for Scientific Research No. 23791737 from the Japan Society for the Promotion of Science.

Received: 15 March 2016 Accepted: 27 October 2016

Published online: 04 November 2016

\section{References}

Chowdhury R, Abbas A, Idriz S, Hoy A, Rutherford EE, Smart JM (2012) Should warfarin or aspirin be stopped prior to prostate biopsy? An analysis of bleeding complications related to increasing sample number regimes. Clin Radiol 67:e64-e70. doi:10.1016/j.crad.2012.08.005

Cochlin DL (2005) Biopsy of the prostate guided by transrectal ultrasound: relation between warfarin use and bleeding complications. Clin Radiol 60:457-458

Connor SE, Wingate JP (1999) Management of patients treated with aspirin or warfarin and evaluation of haemostasis prior to prostatic biopsy: a survey of current practice amongst radiologists and urologists. Clin Radiol 54:598-603

Douketis JD, Berger PB, Dunn AS, Jaffer AK, Spyropoulos AC, Becker RC, Ansell $J$ (2008) The perioperative management of antithrombotic therapy: American College of Chest Physicians Evidence-Based Clinical Practice Guidelines (8th edition). Chest 133:299S-339S. doi:10.1378/chest.08-0675

Douketis J, Bell AD, Eikelboom J, Liew A (2014) Approach to the new oral anticoagulants in family practice: part 2: addressing frequently asked questions. Can Fam Physician 60:997-1001

El-Hakim A, Moussa S (2010) CUA guidelines on prostate biopsy methodology. Can Urol Assoc J 4:89-94

Gage BF, Waterman AD, Shannon W, Boechler M, Rich MW, Radford M (2001) Validation of clinical classification schemes for predicting stroke: results from the National Registry of Atrial Fibrillation. J Am Med Assoc 285:2864-2870

Gage BF, van Walraven C, Pearce L, Hart RG, Koudstaal PJ, Boode BS, Petersen P (2004) Selecting patients with atrial fibrillation for anticoagulation: stroke risk stratification in patients taking aspirin. Circulation 110:2287-2292. doi:10.1161/01.cir.0000145172.55640.93

Garcia DA, Regan S, Henault LE, Upadhyay A, Baker J, Othman M, Hylek EM (2008) Risk of thromboembolism with short-term interruption of warfarin therapy. Arch Intern Med 168:63-69. doi:10.1001/ archinternmed.2007.23

Giannarini G et al (2007) Continuing or discontinuing low-dose aspirin before transrectal prostate biopsy: results of a prospective randomized trial. Urology 70:501-505. doi:10.1016/j.urology.2007.04.016

Ihezue CU, Smart J, Dewbury KC, Mehta R, Burgess L (2005) Biopsy of the prostate guided by transrectal ultrasound: relation between warfarin use and incidence of bleeding complications. Clin Radiol 60:459-463; discussion 457-458. doi:10.1016/j.crad.2004.10.014

Kakehi Y, Naito S (2008) Complication rates of ultrasound-guided prostate biopsy: a nation-wide survey in Japan. Int J Urol 15:319-321. doi:10.1111/j.1442-2042.2008.02048.x

Kearon C, Hirsh J (1997) Management of anticoagulation before and after elective surgery. New Engl J Med 336:1506-1511. doi:10.1056/ nejm199705223362107

Loeb S et al (2013) Systematic review of complications of prostate biopsy. Eur Urol 64:876-892. doi:10.1016/j.eururo.2013.05.049

Maan Z, Cutting CW, Patel U, Kerry S, Pietrzak P, Perry MJ, Kirby RS (2003) Morbidity of transrectal ultrasonography-guided prostate biopsies in patients after the continued use of low-dose aspirin. BJU Int 91:798-800

Murasaki K (2011) Guidelines for management of anticoagulant and antiplatelet therapy in cardiovascular disease (JCS 2009). Nihon Rinsho Jpn J Clin Med 69(Suppl 9):567-571

Pinkhasov Gl et al (2012) Complications following prostate needle biopsy requiring hospital admission or emergency department visitsexperience from 1000 consecutive cases. BJU Int 110:369-374. doi:10.1111/j.1464-410X.2011.10926.x 
Nam RK et al (2013) Increasing hospital admission rates for urological complications after transrectal ultrasound guided prostate biopsy. J Urol 189:S12-S17; discussion S17-S18. doi:10.1016/j.juro.2012.11.015

Ramachandran N, MacKinnon A, Allen C, Dundas D, Patel U (2005) Biopsy of the prostate guided by transrectal ultrasound: relation between warfarin use and incidence of bleeding complications. Clin Radiol 60:1130. doi:10.1016/j.crad.2005.04.017

Rosario DJ et al (2012) Short term outcomes of prostate biopsy in men tested for cancer by prostate specific antigen: prospective evaluation within ProtecT study. BMJ 344:d7894. doi:10.1136/bmj.d7894
Wahl MJ (1998) Dental surgery in anticoagulated patients. Arch Intern Med 158:1610-1616

Wysokinski WE, McBane RD, Daniels PR, Litin SC, Hodge DO, Dowling NF, Heit JA (2008) Periprocedural anticoagulation management of patients with nonvalvular atrial fibrillation. Mayo Clin Proc 83:639-645. doi:10.4065/83.6.639

Yasaka M, Minematsu K, Yamaguchi T (2001) Optimal intensity of international normalized ratio in warfarin therapy for secondary prevention of stroke in patients with non-valvular atrial fibrillation. Int Med 40:1183-1188

\section{Submit your manuscript to a SpringerOpen ${ }^{\odot}$ journal and benefit from:}

- Convenient online submission

- Rigorous peer review

- Immediate publication on acceptance

- Open access: articles freely available online

- High visibility within the field

- Retaining the copyright to your article 